\title{
The Associations of Trajectory of Sleep Duration and Inflammation with Hypertension: A Longitudinal Study in China
}

\author{
Lili Huang ${ }^{1, *}$ \\ Zichong Long ${ }^{1} * *$ \\ Jiajun Lyu' \\ Yiting Chen' \\ Rong $\mathrm{Li}^{\prime}$ \\ Yanlin Wang ${ }^{2}$ \\ Shenghui Li (D) ${ }^{1,3}$
}

'School of Public Health, Shanghai Jiao Tong University School of Medicine, Shanghai, People's Republic of China; ${ }^{2}$ Prenatal Diagnosis Department, International Peace Maternity \& Child Health Hospital, Shanghai Jiao Tong University School of Medicine, Shanghai, People's Republic of China; ${ }^{3} \mathrm{MOE}$ Shanghai Key Laboratory of Children's Environmental Health, Xinhua Hospital, Shanghai Jiao Tong University School of Medicine, Shanghai, People's Republic of China

*These authors contributed equally to this work
Correspondence: Shenghui $\mathrm{Li}$ School of Public Health, Shanghai Jiao Tong University, 227 South Chongqing Road, Huangpu District, Shanghai,

People's Republic of China

$\mathrm{Tel} / \mathrm{Fax}+86-2 \mathrm{I}-63846590$

Email Ish9907@I63.com;

submission9907@I63.com
Background: Existing evidence suggested that sleep duration may be involved in hypertension; however, the conclusions were still controversial. This study aimed to examine the association of longitudinal trajectory of sleep duration with hypertension and to explore the role of the inflammation in such associations.

Methods: A total of 3178 subjects over 30 years of age without hypertension were enrolled in 2004, and they were followed until 2009. Self-reported sleep duration was recorded, and inflammation was measured by highly sensitive $\mathrm{C}$ reactive protein (hs-CRP). Log-binomial regression models were applied to examine the association of sleep duration trajectory and inflammation with the risk of hypertension. The mediating effect of elevated hs-CRP was examined by the bootstrap and the process software.

Results: The prevalence of persistent short ( $\leq 7$ hours/day), normal ( $8-9$ hours/day), and long ( $>9$ hours/day) sleep duration over 5 years were $9.1 \%, 37.7 \%$, and $2.3 \%$, respectively. The incidence of hypertension was $26.6 \%$ during the follow-up period. Compared with those who persistently slept 8-9 hours/day from baseline to follow-up, those who persistently slept $\leq 7$ hours/day, persistently slept $\geq 10$ hours/day, and those whose sleep duration changed have higher risks of hypertension by 1.375 -fold (95\% CI: 1.121, 1.686), 1.557-fold (95\% CI: $1.171,2.069)$ and 1.299 -fold (95\% CI: 1.135, 1.487), respectively. In addition, persistently slept $\leq 7$ hours/day was found to be associated with higher risk of inflammation (RR: 1.285, 95\% CI: 1.008, 1.638). The mediation analysis did not find significant mediating effect of elevated CRP on the association between sleep duration trajectory and hypertension.

Conclusion: Experiencing both a short or long sleep duration, especially for a long time, could lead to higher risk of hypertension. Persistent exposure to short sleep duration was also associated with inflammation. However, the higher risk of hypertension caused by persistent short sleep duration does not seem to be directly mediated through inflammation.

Keywords: sleep duration, trajectory, inflammation, hypertension, longitudinal study

\section{Introduction}

During the past two decades, the prevalence of hypertension has been increasing all over the world. ${ }^{1}$ Due to its great contribution to human morbidity and mortality, hypertension is now a worldwide public health concern. A growing body of studies suggested that sleep duration may be associated with a higher risk of hypertension. $^{2-4}$ However, most existing evidence was mainly based on crosssectional studies; ${ }^{5-7}$ the longitudinal data were limited and controversial. ${ }^{8,9}$ A longitudinal study in China indicated that compared with participants who slept 8 hours/day, those who slept $\leq 7$ hours/day were associated with higher risk of 
hypertension; ${ }^{8}$ however, another longitudinal study conducted in Finland found both short ( $<7$ hours/night) and long ( $\geq 9$ hours/night) sleep duration were not associated with the incidence of hypertension, compared with those who slept $7-8$ hours/night. ${ }^{9}$ Considering sleep is a modifiable behavioral factor, further evidence is warranted on the association between sleep duration and hypertension.

The mechanism between sleep duration and hypertension remains unclear, and the emerging evidence points to the systemic inflammation. Chronic and mild inflammation has been demonstrated to be an important risk factor for the development of metabolic diseases. ${ }^{10,11} \mathrm{C}$-reactive protein (CRP), as a marker of systemic inflammation, was found to be associated with a higher risk of hypertension. ${ }^{12-14}$ An experimental study demonstrated that both total and partial sleep deprivation, even for only one night, could result in elevated CRP. ${ }^{15}$ However, epidemiological surveys provided mixed findings. ${ }^{16,17}$ A national longitudinal study in the US with 6 year follow-up found sleep duration $\leq 6$ hours/ night was associated with elevated CRP $(\geq 3 \mathrm{mg} / \mathrm{dL}) .{ }^{16}$ But data from the UK found that compared with those who slept 7-8 hours, sleep duration $\leq 5,5-6,6-7$ and $>8$ hours was not associated with CRP through a 4-year follow-up. ${ }^{17}$ Therefore, the role of inflammation in the association between sleep and hypertension is far from clear. To our knowledge, till now there was no population-based study yet to explore the role of inflammation in the association between sleep duration and hypertension.

Sleep is a dynamic process throughout an individual's lifespan, which was affected by environmental, physical, and psychological factors. ${ }^{18}$ Recent evidence suggested that changes in sleep duration or persistent short or long sleep duration over time were associated with higher risk of adverse outcomes, ${ }^{19-21}$ which indicated that the trajectory of sleep may be a better predictor of health risk than a single measurement.

In the present study, we particularly focus on the trajectory of sleep duration, to examine its association with hypertension by a 5-year follow-up, which could provide stronger evidence for us to understand the role of sleep duration in the development of hypertension; meanwhile, the potential effect of CRP on the association between sleep duration and hypertension would be specifically explored based on population-based study.

\section{Methods}

\section{Study Design and Participants}

The present study was derived from China Health and Nutrition Survey (CHNS), a nationally ongoing survey based on household since 1989, covering 9 provinces in national wide. The survey was followed every two to four years and the investigation content included personal basic characteristics, lifestyles, family information, physical examination, and clinical detection. Sleep duration data was collected since 2004. The available clinical detection data were only in 2009. Further detailed information can be obtained at http://www.cpc.unc.edu/projects/china. The CHNS has been approved by the institutional review committees of the National Institute of Nutrition and Food Safety, Chinese Center for Disease Control and Prevention, the University of North Carolina at Chapel Hill, and the China-Japan Friendship Hospital, Ministry of Health. All the participants signed informed consent forms at the survey enrollment.

In the present analysis, we targeted the middle-aged and elderly participants who took part in both surveys of 2004 and 2009. Totally, 6341 participants aged $\geq 30$ years in 2004 were followed until 2009. Among them, 827 were excluded due to the missing or extreme data on sleep duration, blood pressure (BP), body mass index (BMI) (the extreme data was defined as beyond three times of the interquartile interval); 987 were excluded because of the missing data on CRP or the value of CRP $>10 \mathrm{mg} / \mathrm{L}$, which was defined as acute inflammation. ${ }^{22}$ Besides, 980 participants who have hypertension at baseline were excluded. Moreover, because of the unavailability of CRP at 2004, 369 participants who have diabetes, obesity or infectious diseases were further excluded to control the possible influence of baseline inflammation. Finally, a total of 3178 participants qualified the inclusion criteria of this study (Figure 1).

\section{Measurements of Sleep Duration and CRP}

Sleep duration was measured through the question "How many hours do you usually sleep each day, including daytime and nighttime in the last year?". Sleep duration was categorized into three groups: $\leq 7,8-9$ and $\geq 10$ hours/day, therefore, the trajectory of sleep duration from 2004 to 2009 were categorized into: $\leq 7$ hours/day $\rightarrow \leq 7$ hours/day as persistent short sleep duration; $\geq 10$ hours/day $\rightarrow \geq 10$ hours/day as persistent long sleep duration; 8-9 hours/ 


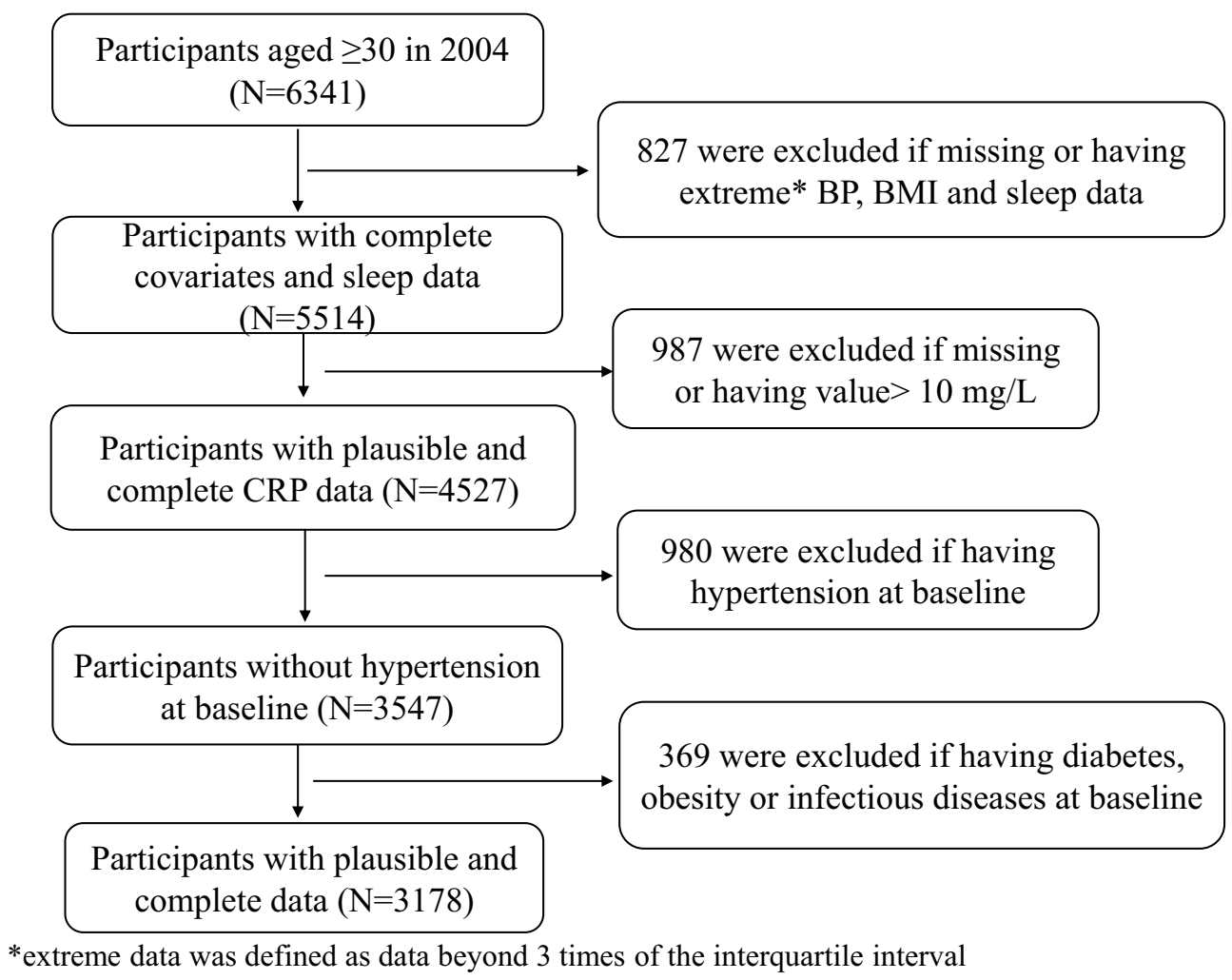

Figure I Sampling flowchart and participants enrollment.

day $\rightarrow 8-9$ hours/day as persistent normal sleep duration; considering the limited sample size, the others, covering sleep duration either decreased or increased, were defined as changed pattern over time.

For each participant, a fasting blood sample was drawn from the antecubital vein. The plasma and serum samples centrifuged from whole blood were frozen and stored, and then were transported to a national lab in Beijing. Highly sensitive $\mathrm{C}$ reactive protein (hs-CRP) was detected using the immunoturbidimetric method (Hitachi 7600 automated analyzer, Hitachi, Tokyo, Japan). Values $>2 \mathrm{mg} / \mathrm{L}$ (elevated hs-CRP) were considered inflammation due to its association with high risk of future cardiovascular events ${ }^{23}$ and which has been recommended by previous study. ${ }^{23}$

\section{Definition of Hypertension}

Hypertension was defined as a self-report of diagnosis by a physician via the question "Has a doctor ever told you that you suffer from high blood pressure?". As for those subjects who did not answer this question or answered "no" or "unknown", their BP values were examined by trained examiners, mercury sphygmomanometers with appropriate-sized cuffs were applied. Measurements were taken in triplicate after at least a 10-min rest, and the three readings were averaged as the $\mathrm{BP}$ values, and hypertension was diagnosed as average systolic BP (SBP)/diastolic BP (DBP) $\geq 140 / 90 \mathrm{mmHg}$.

\section{Covariates}

BMI was calculated as weight in kilograms divided by height in meters squared. Education was categorized into low (less than high school), medium (from high school to technical school), and high (higher than university) level. Smoke (yes or no) was based on the question "Ever smoked cigarettes?" and a smoker was defined as smoked continuously or cumulatively for 6 months or more. Drank alcohol (yes or no) was based on the question "Drank alcohol last year?". Physical activity (PA)/day was based on transportation and leisure activities, and expressed as metabolic equivalent of task (MET) by using a Compendium of Physical Activities. ${ }^{24}$ Sedentary time was estimated based on major screen exposure (watching television, video compact disc, video games, surfing internet, joining chat rooms, and playing computer games) and other sedentary activities. The following covariates were included, which have been demonstrated in published literature to be associated with hypertension: ${ }^{25}$ age, BMI, gender (male vs female), education (low, medium, high), 
family income per capita (tertiles), smoke (yes vs no), drank alcohol (yes vs no), PA (tertiles), sedentary time (tertiles). Models for each outcome accounted for the same covariates to maintain consistency.

\section{Statistical Analysis}

The sample characteristics between 2004 and 2009 were compared, Student's test, Kruskal-Wallis test, and chisquare tests being applied where appropriate. The distributions of elevated hs-CRP and hypertension were analyzed based on sleep duration groups, and the difference was estimated by chi-square tests.

Log-binomial regression was used to estimate the associations of sleep duration with elevated CRP and hypertension, as well as the association of elevated CRP with hypertension, in which the effect was expressed as relative risk (RR). In the crude model, only exposure (sleep duration or elevated CRP) was included. Then, age and gender were further adjusted in the adjusted model 1; education, family income per capita, PA, sedentary time/day, smoke, drank alcohol, and BMI were additionally adjusted in the adjusted model 2 . In order to explore the role of elevated hs-CRP in the association between sleep duration and hypertension, mediation analysis was further conducted, in which elevated hs-CRP was the mediator. The mediating effect was estimated using the bootstrapping method proposed by Preacher and Hayes. ${ }^{26}$

All statistical analyses were conducted by IBM SPSS Statistics (version 24.0, IBM Corp.). Statistical tests were twotailed and were assessed at a $5 \%$ level of significance $(p<0.05)$.

\section{Results}

\section{Characteristics of the Study Population}

The characteristics of participants are presented in Table 1. Of 3178 participants, male was $43.0 \%$; the mean age was 47.98 [standard deviation $(\mathrm{SD})=11.64$ ] years old in 2004 . The mean sleep duration was slightly decreased from $8.13(\mathrm{SD}=1.16)$ hours/day in 2004 to $7.91(\mathrm{SD}=1.22)$ hours/day in 2009. The percentage of sleep duration $\leq 7$ hours was increased from $24.3 \%$ to $30.6 \%$ while sleep duration $\geq 10$ hours was decreased from $13.5 \%$ to $10.7 \%$. The incidence of elevated CRP and hypertension in 2009 was $18.6 \%$ and $26.6 \%$, respectively.

\section{The Distribution of Elevated CRP and Hypertension According to Sleep Duration}

As Table 2 shows, the prevalence of elevated CRP was higher in those who slept $\leq 7$ hours /day and $\geq 10$ hours/day than those who slept 8-9 hours/day, only at follow-up survey $(p=0.024)$. Both at baseline and follow-up survey, the incidence of hypertension was significantly higher in group of sleep duration $\leq 7$ hours /day and sleep duration $\geq 10$ hours/ day than that in sleep duration $8-9$ hours/day $(p \leq 0.001)$. Moreover, the incidence of hypertension was higher in subjects who have persistent short sleep (31.5\%), persistent long sleep $(40.3 \%)$ or changed pattern $(29.1 \%)$ than in those who have persistent normal sleep duration (21.1\%).

\section{The Association of Sleep Duration with Hypertension}

As showed in Table 3, sleep duration $\leq 7$ hours/day and $\geq 10$ hours/day at baseline were associated with a higher risk of hypertension (RR: $1.239,95 \%$ CI: 1.083, 1.418, RR: 1.279 , $95 \%$ CI: $1.086,1.506$, respectively) in crude model. Similarly, such association was also occurred in follow-up survey (RR: 1.252, 95\% CI: 1.102, 1.424; RR: 1.417, 95\% CI: $1.192,1.685$, respectively). For changes in sleep duration, compared with persistently slept $8-9$ hours/day, persistently slept $\leq 7$ hours/day, $\geq 10$ hours/day and changed pattern over the 5 years showed a 1.477 -fold (95\% CI: 1.201 , 1.817), 1.949-fold (95\% CI: 1.442, 2.634) and 1.405-fold (95\% CI: 1.228, 1.609) higher risk of hypertension, respectively, in crude model. The effects were somewhat attenuated but still kept significance after adjusting for age and gender. We further adjusted lifestyles and BMI, sleep duration $\leq 7$ hours/day and $\geq 10$ hours/day were still associated with higher risk of hypertension (RR: 1.192, 95\% CI: 1.045, 1.359, RR: $1.169,95 \%$ CI: $0.996,1.371$, respectively) at baseline and (RR: 1.199, 95\% CI: 1.059, 1.359, RR: 1.205, 95\% CI: 1.019, 1.424, respectively) at follow-up survey. Similarly, persistently slept $\leq 7$ hours/day, $\geq 10$ hours/day and changed pattern over the 5 years were also presented 1.375-fold (95\% CI: 1.121, 1.686), 1.557-fold (95\% CI: $1.171,2.069)$ and 1.299 -fold (95\% CI: $1.135,1.487)$ higher risk of hypertension, respectively.

\section{The Association of Sleep Duration with Elevated CRP}

The association between sleep duration and elevated CRP is presented in Table 4, elevated hs-CRP was not demonstrated to be associated with sleep duration at baseline. However, sleep duration $\leq 7$ hours/day at follow-up survey increased the risk of elevated hs-CRP (RR: $1.223,95 \% \mathrm{CI}$ : $1.042,1.436$ ), so did persistent sleep duration $\leq 7$ hours/ day (RR: 1.348, 95\% CI: 1.060, 1.714) in crude model. 
Table I Sample Characteristics in 2004 and 2009

\begin{tabular}{|c|c|c|c|}
\hline & 2004 & 2009 & \multirow[t]{2}{*}{$p$} \\
\hline & $\begin{array}{c}\text { Mean (SD)/n } \\
(\%)\end{array}$ & $\begin{array}{c}\text { Mean (SD)/n } \\
(\%)\end{array}$ & \\
\hline \multicolumn{4}{|l|}{ Personal characteristics } \\
\hline Energy intake (kcal/d) & $2257.67(649.36)$ & $2158.63(639.79)$ & $<0.001$ \\
\hline BMI $\left(\mathrm{kg} / \mathrm{m}^{2}\right)$ & $22.44(2.57)$ & $22.81(2.84)$ & $<0.001$ \\
\hline \multicolumn{4}{|l|}{ Gender } \\
\hline Male & $1366(43.0)$ & - & \\
\hline Female & $1812(57.0)$ & - & \\
\hline Age (years) & $47.98(11.64)$ & $52.95(11.64)$ & $<0.001$ \\
\hline $30-45$ & $1329(4 \mid .8)$ & $851(26.8)$ & $<0.001$ \\
\hline $45-60$ & $1316(41.4)$ & $1469(46.2)$ & \\
\hline $60-75$ & $482(15.2)$ & $712(22.4)$ & \\
\hline$\geq 75$ & $51(1.6)$ & $146(4.6)$ & \\
\hline \multicolumn{4}{|l|}{ Residence } \\
\hline Urban & $939(29.5)$ & - & \\
\hline Rural & $2239(70.5)$ & - & \\
\hline \multicolumn{4}{|l|}{ Education } \\
\hline Low & 2538 (79.9) & - & \\
\hline Medium & $533(16.8)$ & - & \\
\hline High & $105(3.3)$ & - & \\
\hline \multicolumn{4}{|l|}{ Income } \\
\hline Low & $1048(33.1)$ & $1036(33.4)$ & 0.439 \\
\hline Medium & $1098(34.7)$ & $1034(33.3)$ & \\
\hline High & $1017(32.2)$ & $1036(33.4)$ & \\
\hline \multicolumn{4}{|l|}{ Physical activities } \\
\hline $\mathrm{MET} /$ day & $1.59(7.92)$ & $1.59(6.59)$ & 0.970 \\
\hline Low & $1673(52.6)$ & $1674(52.7)$ & $<0.001$ \\
\hline Medium & $874(27.5)$ & $798(25.1)$ & \\
\hline High & $631(19.9)$ & $706(22.2)$ & \\
\hline \multicolumn{4}{|l|}{$\begin{array}{l}\text { Sedentary time (hours/ } \\
\text { day) }\end{array}$} \\
\hline Low & $1057(33.3)$ & $995(31.3)$ & 0.216 \\
\hline Medium & $1016(31.8)$ & $1017(32.0)$ & \\
\hline High & $1105(34.9)$ & $1166(36.7)$ & \\
\hline \multicolumn{4}{|l|}{ Smoke } \\
\hline No & $2163(68.2)$ & $2206(69.5)$ & 0.275 \\
\hline Yes & $1009(31.8)$ & $970(30.5)$ & \\
\hline \multicolumn{4}{|l|}{ Drank alcohol } \\
\hline No & $2164(68.2)$ & $2178(68.6)$ & 0.789 \\
\hline Yes & $1007(31.8)$ & $999(31.4)$ & \\
\hline \multicolumn{4}{|c|}{ Sleep duration, CRP, and hypertension } \\
\hline Sleep duration (hours/day) & $8.13(1.16)$ & $7.91(1.22)$ & $<0.001$ \\
\hline$\leq 7$ hours & $773(24.3)$ & $973(30.6)$ & $<0.001$ \\
\hline $8-9$ hours & $1977(62.2)$ & $1866(58.7)$ & \\
\hline$\geq 10$ hours & $428(13.5)$ & $339(10.7)$ & \\
\hline $\mathrm{CRP}(\mathrm{mg} / \mathrm{L})$ & - & $1.0(0.0,2.0)$ & \\
\hline
\end{tabular}

(Continued)
Table I (Continued).

\begin{tabular}{|l|c|c|c|}
\hline & $\mathbf{2 0 0 4}$ & $\mathbf{2 0 0 9}$ & \multirow{2}{*}{} \\
\cline { 2 - 3 } & $\begin{array}{c}\text { Mean (SD)/n } \\
\text { (\%) }\end{array}$ & $\begin{array}{c}\text { Mean (SD)/n } \\
\text { (\%) }\end{array}$ & \\
\hline Elevated CRP & & & \\
No & - & $2588(81.4)$ & \\
Yes & - & $590(18.6)$ & \\
SBP & $115.34(11.25)$ & $122.23(16.37)$ & \\
DBP & $74.72(7.97)$ & $79.75(10.79)$ & \\
\hline Hypertension & & & \\
No & - & $2334(73.4)$ & \\
Yes & - & $844(26.6)$ & \\
\hline
\end{tabular}

Abbreviations: SD, standard deviation; BMI, body mass index; CRP, C-reactive protein; SBP, systolic blood pressure; DBP, diastolic blood pressure; MET, metabolic equivalent.

The estimated effect of sleep duration $\leq 7$ hours/day on elevated hs-CRP was attenuated (RR: 1.182, 95\% CI: $1.007,1.388)$ at follow-up survey after adjusting for age and gender, as well as the effect of persistently slept $\leq 7$ hours/day (RR: 1.283, 95\% CI: 1.006, 1.635). When additionally adjusted for lifestyles and BMI, the significant effect still kept, only in persistent sleep duration $\leq 7$ hours/day (RR: 1.285, 95\% CI: 1.008, 1.638). No significant association was found between long sleep duration and elevated CRP.

\section{Mediation Analysis}

The association of elevated CRP with hypertension was also examined, and we found that the elevated CRP was associated with higher risk of hypertension (RR: 1.204, 95\% CI: $1.059,1.368)$ even adjusted for multiple covariates (Table 5). Further, mediation analysis was conducted to evaluate the mediating role of elevated hs-CRP in the association between sleep trajectory and hypertension. However, the mediating effect was not found to be significant (Table S1).

\section{Discussion}

In this study, we explored the impact of sleep duration as well as its long-time trajectory on the risk of hypertension, and examined the role of inflammation in such association. Our findings revealed that for adults who persistently keep a short or long sleep duration, and those whose sleep duration has changed during the follow-up periods, the risk of hypertension has been increased. Besides, the association of persistent short sleep duration with elevated CRP was also observed. However, mediation analysis did 
Table 2 The Distribution of Elevated CRP and Hypertension According to Sleep Duration Categories

\begin{tabular}{|c|c|c|c|c|c|}
\hline & $\mathbf{N}$ & Elevated CRP (n, \%) & $p$ & Hypertension (n, \%) & $p$ \\
\hline \multicolumn{6}{|l|}{ Participants at 2004} \\
\hline$\leq 7$ hours & $773(24.3)$ & I44 (I8.6) & 0.834 & $235(30.4)$ & 0.001 \\
\hline $8-9$ hours & $1977(62.2)$ & $371(18.8)$ & & $479(24.2)$ & \\
\hline$\geq 10$ hours & $428(13.5)$ & $75(17.5)$ & & $130(30.4)$ & \\
\hline \multicolumn{6}{|l|}{ Participants at 2009} \\
\hline$\leq 7$ hours & $973(30.6)$ & $202(20.8)$ & 0.024 & $286(29.4)$ & $<0.001$ \\
\hline $8-9$ hours & I866 (58.7) & $317(17.0)$ & & $445(23.8)$ & \\
\hline$\geq 10$ hours & $339(10.7)$ & 7I (20.9) & & $113(33.3)$ & \\
\hline \multirow{2}{*}{\multicolumn{6}{|c|}{$\begin{array}{l}\text { Sleep change from } 2004 \text { to } \\
2009\end{array}$}} \\
\hline & & & & & \\
\hline$\leq 7$ hours $/$ day $\rightarrow \leq 7$ hours & $289(9.1)$ & $70(24.2)$ & 0.054 & $91(31.5)$ & $<0.001$ \\
\hline$\geq 10$ hours $\rightarrow \geq 10$ hours & $72(2.3)$ & $16(22.2)$ & & $29(40.3)$ & \\
\hline Changed pattern & $1620(50.9)$ & $288(17.8)$ & & $472(29.1)$ & \\
\hline $8-9$ hours $\rightarrow 8-9$ hours & II 97 (37.7) & $216(18.0)$ & & $252(2 I . I)$ & \\
\hline
\end{tabular}

Table 3 The Association of Sleep Duration with Hypertension

\begin{tabular}{|c|c|c|c|c|}
\hline & \multirow[t]{2}{*}{ n (\%) } & Crude Model & Adjusted Model I & Adjusted Model 2 \\
\hline & & $\operatorname{RR}(95 \% \mathrm{Cl})$ & $\operatorname{RR}(95 \% \mathrm{Cl})$ & $\mathbf{R R}(95 \% \mathrm{Cl})$ \\
\hline \multicolumn{5}{|l|}{2004} \\
\hline$\leq 7$ hours & $773(24.3)$ & $1.239(1.083,1.418)$ & I.I $178(1.032,1.344)$ & I.192 (I.045, I.359) \\
\hline $8-9$ hours & $1977(62.2)$ & Reference & Reference & Reference \\
\hline$\geq 10$ hours & $428(13.5)$ & $1.279(1.086,1.506)$ & I.I79 (I.005, I.383) & $1.169(0.996,1.37$ I $)$ \\
\hline \multicolumn{5}{|l|}{2009} \\
\hline$\leq 7$ hours & $973(30.6)$ & $1.252(1.102,1.424)$ & $1.197(1.057,1.356)$ & $1.199(1.059,1.359)$ \\
\hline $8-9$ hours & $1866(58.7)$ & Reference & Reference & Reference \\
\hline$\geq 10$ hours & $339(10.7)$ & $1.417(1.192,1.685)$ & $1.220(1.03 \mathrm{I}, 1.443)$ & $1.205(1.019,1.424)$ \\
\hline \multicolumn{5}{|c|}{ Sleep change from 2004 to 2009} \\
\hline$\leq 7$ hours $/$ day $\rightarrow \leq 7$ hours & $289(9.1)$ & $\mathrm{I} .477$ (I.20I, I.8I7) & $1.383(1.128,1.696)$ & $1.375(1.121,1.686)$ \\
\hline$\geq 10$ hours $\rightarrow \geq 10$ hours & $72(2.3)$ & $1.949(1.442,2.634)$ & $1.580(1.185,2.108)$ & $1.557(1.171,2.069)$ \\
\hline Changed pattern & $1620(50.9)$ & $1.405(1.228,1.609)$ & $1.332(1.164,1.524)$ & $1.299(\mathrm{I} .135, \mathrm{I} .487)$ \\
\hline $8-9$ hours $\rightarrow 8-9$ hours & II $97(37.7)$ & Reference & Reference & Reference \\
\hline
\end{tabular}

Notes: Adjusted Model I adjusted age, gender. Adjusted Model 2 additionally adjusted education, family income per capita, physical activities, sedentary time per day, smoke, drank alcohol, BMI.

not find elevated CRP to be a significant mediator in the associations between persistent short sleep duration and hypertension.

Compelling evidence have demonstrated that sleep duration may be involved in the development of hypertension; however, consistent conclusion has not yet been fully achieved. Majority of studies have established the relationship between short sleep duration and subsequent higher risk of hypertension, ${ }^{27,28}$ which is consistent with our findings. Meanwhile, our study also found long sleep duration was associated with higher risk of hypertension, which was an "U-shaped" association. Similarly, another study conducted in China observed that sleep duration $<7$ and $\geq 9$ hours/night were associated with high BP (SBP $\geq 130$ and/or DBP $\geq 85 \mathrm{mmHg}$ or diagnosed hypertension) among females; ${ }^{29}$ and a study conducted in US also found that sleep duration $<7$ and $\geq 10$ hours/day were associated with higher risk of hypertension, ${ }^{30}$ which suggests a normal sleep duration may be essential to maintain relatively low risk of hypertension. However, the 
Table 4 The Association of Sleep Duration with Elevated CRP

\begin{tabular}{|c|c|c|c|}
\hline & Crude Model & Adjusted Model I & Adjusted Model 2 \\
\hline & $\mathbf{R R}(95 \% \mathrm{CI})$ & RR (95\% CI) & RR (95\% CI) \\
\hline \multicolumn{4}{|l|}{2004} \\
\hline$\leq 7$ hours & $0.998(0.837,1.190)$ & $0.969(0.812,1.156)$ & $0.959(0.806,1.141)$ \\
\hline $8-9$ hours & Reference & Reference & Reference \\
\hline$\geq 10$ hours & $0.937(0.746,1.177)$ & $0.908(0.722,1.142)$ & 0.88 I $(0.702,1.105)$ \\
\hline \multicolumn{4}{|l|}{2009} \\
\hline$\leq 7$ hours & $1.223(1.042,1.436)$ & I.I82 (1.007, I.388) & I.I $69(0.996,1.372)$ \\
\hline $8-9$ hours & Reference & Reference & Reference \\
\hline$\geq 10$ hours & $1.220(0.956,1.543)$ & $1.130(0.892,1.431)$ & $1.110(0.878,1.402)$ \\
\hline \multicolumn{4}{|c|}{ Sleep change from 2004 to 2009} \\
\hline$\leq 7$ hours $/$ day $\rightarrow \leq 7$ hours & $1.348(1.060,1.714)$ & $1.283(1.006,1.635)$ & $1.285(1.008,1.638)$ \\
\hline$\geq 10$ hours $\rightarrow \geq 10$ hours & $1.255(0.802,1.965)$ & $1.166(0.742,1.832)$ & $1.201(0.765,1.886)$ \\
\hline Changed pattern & 1.001 (0.85I, I.I77) & $0.977(0.830,1.150)$ & $0.965(0.821,1.134)$ \\
\hline $8-9$ hours $\rightarrow 8-9$ hours & Reference & Reference & Reference \\
\hline
\end{tabular}

Notes: Adjusted Model I adjusted age, gender. Adjusted Model 2 additionally adjusted education, family income per capita, physical activities, sedentary time per day, smoke, drank alcohol, BMI.

Table 5 The Association of hs-CRP with Hypertension

\begin{tabular}{|l|l|l|l|}
\hline & Crude Model & Adjusted Model I & Adjusted Model 2 \\
\hline hs-CRP (mg/L) & RR (95\% Cl) & RR (95\% Cl) & RR (95\% CI) \\
\hline$\leq 2$ & Reference & Reference & Reference \\
$>2$ & $1.380(1.21 \mathrm{I}, \mathrm{I.572})$ & $1.235(\mathrm{I} .088,1.402)$ & $1.204(\mathrm{I}) .059,1.368)$ \\
\hline
\end{tabular}

Notes: Adjusted Model I adjusted age, gender. Adjusted Model 2 additionally adjusted education, family income per capita, physical activities, sedentary time per day, smoke, drank alcohol, BMI.

differences in the sample characteristics, definition of short and long sleep duration, as well as covariate controlling strategy, could result in the inconsistence of the findings. ${ }^{9,31}$

In addition, the sleep duration fluctuates day from day, contingency is inevitable when adopting one-off measurement, while the changes in sleep duration over time could provide deeper and more reliable insight on its health impact. $^{20,21}$ To date, the evidence on the association of sleep duration changes with the risk of hypertension was quite scarce, with only several studies focused on the topic. A study revealed that persistently slept $\leq 6$ hours, compared with persistently slept 7 hours, increased the risk of hypertension among women in Korea through over 5 years follow-up; ${ }^{20}$ another study conducted in China suggested persistently decrease of sleep duration was significantly associated with an increased risk of hypertension. ${ }^{32}$ In consistent with the two findings, our results also observed that persistent short sleep duration increased the risk of hypertension. In addition, our findings found that persistently slept $\geq 10$ hours/day and changed pattern over time also increased the risk of hypertension. Thus, keeping normal sleep duration over time, rather than only one-off measurement, should be emphasized in further perspective studies.

A bulk of literature have reported the association of sleep duration with inflammation, ${ }^{33-35}$ however, the evidence from prospective study was limited. A cohort study among participants aged 18 to 30 years in the US showed short sleep duration ( $<6$ hours) at baseline was associated with an increased level of CRP at follow-up survey; however, the effect became non-significant after adjusting for multi-covariates. ${ }^{36}$ Another prospective cohort study conducted in England did not find the relationship between sleep duration at baseline and follow-up CRP among people aged 50 years and older. ${ }^{17}$ Our analysis was conducted among middle-aged and older participants in China, and we did not observe the sleep duration at baseline was 
associated with an elevated CRP at follow-up, while a significant association was observed between persistent short sleep and elevated CRP. To date, only several studies explored the association of changes in sleep duration with inflammation. Whitehall II study was conducted on participants aged 33-55 years from multicenter in London, demonstrating that decreased sleep duration between two waves of measurements was associated with an increased level of CRP though the significance was disappeared after adjusting for confounders. ${ }^{37}$ Another cohort study was performed among American participants aged 18-26 years and followed up until they were 24-32 years old, and the finding revealed that short sleep duration was associated with higher risk of elevated CRP $(>3 \mathrm{mg} / \mathrm{L}) .{ }^{16}$ Such effect is biologically plausible. The existing data indicated that the hypothalamic-pituitary-adrenal axis (HPA axis) and the sympathetic nervous system (SNS) are the major effector systems involved in sleep. ${ }^{38}$ Sleep deprivation could activate the HPA system to release excessive cortisol, and further induce inflammation by increasing glucocorticoid resistance of immune cells. ${ }^{39,40}$ The activity of SNS could significantly increases when sleep deprivation occurs, ${ }^{41}$ which was implicated in the promoted expression of an inflammatory transcriptional profile. ${ }^{42-44}$

Evidence has suggested that inflammation was implicated in hypertension, ${ }^{45-47}$ which was also observed in our study. Therefore, we conducted mediation analysis to explore the mediating effect of inflammation measured by elevated CRP in the associations between sleep duration trajectory and hypertension; however, in contrast to our expectation, the mediating effect was not significant. Nevertheless, our results should be interpreted with caution, because in all observational studies, the potential assumptions of causality between each pair of factors in mediation analysis were unable to be confirmed. ${ }^{48}$ More studies are needed to clarify the association between short sleep, inflammation, and hypertension.

Our study also found that persistent long sleep duration, in addition to single-point, was associated with hypertension. Similarly, previous studies also reported sleep duration increased $\geq 2$ hours over time or persistent long sleep duration could increase the risk of diabetes ${ }^{21,49}$ and stroke. ${ }^{50}$ Persistent long sleep duration in this study was not associated with inflammation, which differed from persistent short sleep duration. Inflammation in this study may not interpret the effect of long sleep duration on hypertension. The mechanism of the association between long sleep duration and hypertension was still unclear. Several possible pathways may give some explanations. Long sleep duration could reflect the existence of comorbidities, which may lead to sleep, ${ }^{21}$ besides, it may indicate a sedentary lifestyle, which is related to hypertension; ${ }^{51}$ further, long sleep duration was also related to other risk factors of hypertension, such as depression, low socioeconomic status. ${ }^{52}$ More studies are needed to explore the possible mechanisms.

This study has several limitations. Firstly, the sample size was not large enough to categorize sleep duration trajectories into more groups, which restricted the further exploration. Secondly, sleep duration was self-reported but not objective measurements (such as actigraphy); however, it was particularly suitable in large epidemiology studies for its good feasibility and cost-effective features. Fortunately, a previous study has confirmed a moderate correlation between objective and subjective measurements. ${ }^{53}$ Moreover, only information on sleep duration was collected, sleep habits or sleep disturbance (such as sleep apnea) were unavailable, which may be related to the risk of hypertension. ${ }^{54}$ Thirdly, inflammation in this study was measured by CRP; however, other inflammatory markers, such as interleukin-6, tumor necrosis factor- $\alpha$, were also related to sleep duration and hypertension, ${ }^{34,55}$ further studies are needed to estimate the role of these inflammatory markers on the association between sleep duration and hypertension. Finally, our data are based on sleep duration measured at two time points 5 years apart; therefore, we could not capture the full change of sleep duration throughout the 5-year followup period.

\section{Conclusion}

In this study, we found that sleep duration trajectory, including persistent short and long sleep duration, as well as changed pattern over time, could increase higher risk of hypertension. Keeping normal sleep duration over time, rather than only at one moment, should be emphasized in further perspective studies. In addition, persistent short sleep duration also increased the risk of inflammation, highlighting the significance of keeping normal sleep duration to control inflammation. However, we did not observe that CRP mediated the association of persistent short sleep duration with hypertension. Additional studies should be performed to elucidate the mediating roles of CRP and other inflammatory markers. 


\section{Acknowledgments}

The present study derived data from the CHNS. We all thank to the National Institute of Nutrition and Food Safety, Chinese Center for Disease Control and Prevention; the Carolina Population Center, the University of North Carolina at Chapel Hill, the National Institutes of Health, and the Fogarty International Center for the CHNS data collection and analysis since 1989. We also thank the collaboration of the China-Japan Friendship Hospital, and Ministry of Health.

\section{Funding}

The study was funded by grants from National Natural Science Foundation of China $(81874266,81673183)$.

\section{Disclosure}

The authors declare that they have no conflicts of interest.

\section{References}

1. Kearney PM, Whelton M, Reynolds K, Muntner P, Whelton PK, He J. Global burden of hypertension: analysis of worldwide data. Lancet. 2005;365(9455):217-223. doi:10.1016/s0140-6736(05)17741-1

2. Wang Y, Mei H, Jiang YR, et al. Relationship between duration of sleep and hypertension in adults: a meta-analysis. $J$ Clin Sleep Med 2015;11(9):1047-1056. doi:10.5664/jcsm.5024

3. Makarem N, Shechter A, Carnethon MR, et al. Sleep duration and blood pressure: recent advances and future directions. Curr Hypertens Rep. 2019;21(5):33. doi:10.1007/s11906-019-0938-7

4. Guo XF, Zheng LQ, Wang J, Zhang XY, Li J, Sun YX. Epidemiological evidence for the link between sleep duration and high blood pressure: a systematic review and meta-analysis. Sleep Med. 2013;14(4):324-332. doi:10.1016/j.sleep.2012.12.001

5. Matsumoto T, Murase K, Tabara Y, et al. Impact of sleep characteristics and obesity on diabetes and hypertension across genders and menopausal status: the Nagahama study. Sleep. 2018;41(7). doi:10.1093/sleep/zsy071

6. Reis C, Dias S, Rodrigues AM, et al. Sleep duration, lifestyles and chronic diseases: a cross-sectional population-based study. Sleep Sci. 2018;11(4):217-230. doi:10.5935/1984-0063.20180036

7. Sun XM, Yao S, Hu SJ, et al. Short sleep duration is associated with increased risk of pre-hypertension and hypertension in Chinese early middle-aged females. Sleep Breath. 2016;20(4):1355-1362. doi:10.1007/s11325-016-1392-2

8. Feng X, Liu Q, Li Y, et al. Longitudinal study of the relationship between sleep duration and hypertension in Chinese adult residents (CHNS 2004-2011). Sleep Med. 2019;58:88-92. doi:10.1016/j. sleep.2019.01.006

9. Clark AJ, Salo P, Lange T, et al. Onset of impaired sleep and cardiovascular disease risk factors: a longitudinal study. Sleep 2016;39(9):1709-1718. doi:10.5665/sleep.6098

10. Buckley DI, Fu R, Freeman M, Rogers K, Helfand M. C-reactive protein as a risk factor for coronary heart disease: a systematic review and meta-analyses for the U.S. Preventive Services Task Force. Ann Intern Med. 2009;151(7):483-495. doi:10.7326/0003-4819-1517-200910060-00009
11. Kaptoge S, Di Angelantonio E, Lowe G, et al. C-reactive protein concentration and risk of coronary heart disease, stroke, and mortality: an individual participant meta-analysis. Lancet. 2010;375 (9709):132-140. doi:10.1016/s0140-6736(09)61717-7

12. Ridker PM, Buring JE, Cook NR, Rifai N. C-reactive protein, the metabolic syndrome, and risk of incident cardiovascular events: an 8-year follow-up of 14719 initially healthy American women. Circulation. 2003;107(3):391-397. doi:10.1161/01.cir.00000550 14.62083 .05

13. Sung KC, Suh JY, Kim BS, et al. High sensitivity C-reactive protein as an independent risk factor for essential hypertension. $A m$ $J$ Hypertens. 2003;16(6):429-433. doi:10.1016/s0895-7061(03) 00566-1

14. Sesso HD, Buring JE, Rifai N, Blake GJ, Gaziano JM, Ridker PM. $\mathrm{C}$-reactive protein and the risk of developing hypertension. JAMA. 2003;290(22):2945-2951. doi:10.1001/jama.290.22.2945

15. Meier-Ewert HK, Ridker PM, Rifai N, et al. Effect of sleep loss on C-reactive protein, an inflammatory marker of cardiovascular risk. $J$ Am Coll Cardiol. 2004;43(4):678-683. doi:10.1016/j.jacc.20 03.07.050

16. Bakour C, Schwartz S, O’Rourke K, et al. Sleep duration trajectories and systemic inflammation in young adults: results from the National Longitudinal Study of Adolescent to Adult Health (Add Health). Sleep. 2017;40(11). doi:10.1093/sleep/zsx156

17. Jackowska M, Steptoe A. Sleep and future cardiovascular risk: prospective analysis from the English Longitudinal Study of Ageing. Sleep Med. 2015;16(6):768-774. doi:10.1016/j.sleep.2015.02.530

18. Zomers ML, Hulsegge G, van Oostrom SH, Proper KI, Verschuren WMM, Picavet HSJ. Characterizing adult sleep behavior over 20 years-the population-based Doetinchem Cohort Study. Sleep. 2017;40(7). doi:10.1093/sleep/zsx085

19. Ferrie JE, Shipley MJ, Cappuccio FP, et al. A prospective study of change in sleep duration: associations with mortality in the Whitehall II cohort. Sleep. 2007;30(12):1659-1666. doi:10.1093/sleep/ 30.12.1659

20. Kim CW, Chang Y, Kang JG, Ryu S. Changes in sleep duration and subsequent risk of hypertension in healthy adults. Sleep. 2018;41(11). doi:10.1093/sleep/zsy159

21. Ferrie JE, Kivimäki M, Akbaraly TN, et al. Change in sleep duration and type 2 diabetes: the Whitehall II Study. Diabetes Care. 2015;38 (8):1467-1472. doi:10.2337/dc15-0186

22. Pearson TA, Mensah GA, Hong Y, Smith SC Jr. CDC/AHA workshop on markers of inflammation and cardiovascular disease: application to clinical and public health practice: overview. Circulation. 2004;110(25):e543-4. doi:10.1161/01.Cir.0000148979.11121.6b

23. Ye X, Yu Z, Li H, Franco OH, Liu Y, Lin X. Distributions of $\mathrm{C}$-reactive protein and its association with metabolic syndrome in middle-aged and older Chinese people. J Am Coll Cardiol. 2007;49 (17):1798-1805. doi:10.1016/j.jacc.2007.01.065

24. Ainsworth BE, Haskell WL, Whitt MC, et al. Compendium of physical activities: an update of activity codes and MET intensities. Med Sci Sports Exerc. 2000;32(9 Suppl):S498-S504. doi:10.1097/ 00005768-200009001-00009

25. van Oort S, Beulens JWJ, van Ballegooijen AJ, Grobbee DE, Larsson SC. Association of cardiovascular risk factors and lifestyle behaviors with hypertension: a Mendelian Randomization Study. Hypertension. 2020;76(6):1971-1979. doi:10.1161/HYPERTENS IONAHA.120.15761

26. Valeri L, Vanderweele TJ. Mediation analysis allowing for exposure-mediator interactions and causal interpretation: theoretical assumptions and implementation with SAS and SPSS macros [published correction appears in Psychol Methods. 2013 Dec;18(4):474]. Psychol Methods. 2013;18(2):137-150. doi:10.1037/a0031034 
27. Wu X, Sun Y, Niu K, et al. Association of self-reported sleep duration and hypertension: results of a Chinese prospective cohort study. Clin Exp Hypertens. 2016;38(6):514-519. doi:10.3109/10641963.2016.1163367

28. Deng HB, Tam T, Zee BC, et al. Short sleep duration increases metabolic impact in healthy adults: a population-based cohort study. Sleep. 2017;40(10). doi:10.1093/sleep/zsx130

29. Wang H, Zee P, Reid K, et al. Gender-specific association of sleep duration with blood pressure in rural Chinese adults. Sleep Med. 2011;12(7):693-699. doi:10.1016/j.sleep.2010.12.019

30. Grandner M, Mullington JM, Hashmi SD, Redeker NS, Watson NF, Morgenthaler TI. Sleep duration and hypertension: analysis of > 700,000 adults by age and sex. J Clin Sleep Med. 2018;14 (6):1031-1039. doi:10.5664/jcsm.7176

31. Wang D, Zhou Y, Guo Y, et al. The effect of sleep duration and sleep quality on hypertension in middle-aged and older Chinese: the Dongfeng-Tongji Cohort Study. Sleep Med. 2017;40:78-83. doi:10.1016/j.sleep.2017.09.024

32. Wang Y, Hou W, Siddiqi SM, Sun C, Han T, Yang J. Association of sleep trajectory in adulthood with risk of hypertension and its related risk factors: the China Health and Nutrition Survey. J Clin Sleep Med. 2020;16(4):515-521. doi:10.5664/jcsm.8254

33. Simpson N, Dinges DF. Sleep and inflammation. Nutr Rev. 2007;65 (12 Pt 2):S244-52. doi:10.1111/j.1753-4887.2007.tb00371.x

34. Irwin MR, Olmstead R, Carroll JE. Sleep disturbance, sleep duration, and inflammation: a systematic review and meta-analysis of cohort studies and experimental sleep deprivation. Biol Psychiatry. 2016;80 (1):40-52. doi:10.1016/j.biopsych.2015.05.014

35. Dolsen MR, Crosswell AD, Prather AA. Links between stress, sleep, and inflammation: are there sex differences? Curr Psychiatry Rep. 2019;21(2):8. doi:10.1007/s11920-019-0993-4

36. Cho HJ, Seeman TE, Kiefe CI, Lauderdale DS, Irwin MR. Sleep disturbance and longitudinal risk of inflammation: moderating influences of social integration and social isolation in the Coronary Artery Risk Development in Young Adults (CARDIA) study. Brain Behav Immun. 2015;46:319-326. doi:10.1016/j.bbi.2015.02.023

37. Ferrie JE, Kivimäki M, Akbaraly TN, et al. Associations between change in sleep duration and inflammation: findings on C-reactive protein and interleukin 6 in the Whitehall II Study. Am J Epidemiol. 2013;178(6):956-961. doi:10.1093/aje/kwt072

38. Irwin MR. Sleep and inflammation: partners in sickness and in health. Nat Rev Immunol. 2019;19(11):702-715. doi:10.1038/s41577-0190190-z

39. Li LB, Goleva E, Hall CF, Ou LS, Leung DY. Superantigen-induced corticosteroid resistance of human $\mathrm{T}$ cells occurs through activation of the mitogen-activated protein kinase kinase/extracellular signal-regulated kinase (MEK-ERK) pathway. $J$ Allergy Clin Immunol. 2004;114(5):1059-1069. doi:10.1016/j.jaci.2004.08.005

40. Miller AH, Raison CL. The role of inflammation in depression: from evolutionary imperative to modern treatment target. Nat Rev Immunol. 2016;16(1):22-34. doi:10.1038/nri.2015.5
41. Irwin M, Thompson J, Miller C, Gillin JC, Ziegler M. Effects of sleep and sleep deprivation on catecholamine and interleukin-2 levels in humans: clinical implications. J Clin Endocrinol Metab. 1999;84 (6):1979-1985. doi:10.1210/jcem.84.6.5788

42. Irwin MR, Cole SW. Reciprocal regulation of the neural and innate immune systems. Nat Rev Immunol. 2011;11(9):625-632. doi: $10.1038 /$ nri3042

43. Trinder J, Kleiman J, Carrington M, et al. Autonomic activity during human sleep as a function of time and sleep stage. J Sleep Res. 2001;10(4):253-264. doi:10.1046/j.1365-2869.2001.00263.x

44. Palma JA, Urrestarazu E, Lopez-Azcarate J, et al. Increased sympathetic and decreased parasympathetic cardiac tone in patients with sleep related alveolar hypoventilation. Sleep. 2013;36(6):933-940. doi: $10.5665 /$ sleep. 2728

45. De Miguel C, Rudemiller NP, Abais JM, Mattson DL. Inflammation and hypertension: new understandings and potential therapeutic targets. Curr Hypertens Rep. 2015;17(1):507. doi:10.1007/s11906-014-0507-z

46. Agita A, Alsagaff MT. Inflammation, Immunity, and Hypertension. Acta Med Indones. 2017;49(2):158-165.

47. Jayedi A, Rahimi K, Bautista LE, Nazarzadeh M, Zargar MS, ShabBidar S. Inflammation markers and risk of developing hypertension: a meta-analysis of cohort studies. Heart. 2019;105(9):686-692. doi:10.1136/heartjnl-2018-314216

48. Pieters N, Janssen BG, Dewitte H, et al. Biomolecular markers within the core axis of aging and particulate air pollution exposure in the elderly: a cross-sectional study. Environ Health Perspec. 2016;124 (7):943-950. doi:10.1289/ehp.1509728

49. Cespedes EM, Bhupathiraju SN, Li Y, Rosner B, Redline S, Hu FB. Longterm changes in sleep duration, energy balance and risk of type 2 diabetes. Diabetologia. 2016;59(1):101-109. doi:10.1007/s00125-015-3775-5

50. Zhou L, Yu K, Yang L, et al. Sleep duration, midday napping, and sleep quality and incident stroke: the Dongfeng-Tongji cohort. Neurology. 2020;94(4):e345-e356. doi:10.1212/WNL.0000000000008739

51. Lakerveld J, Mackenbach JD, Horvath E, et al. The relation between sleep duration and sedentary behaviours in European adults. Obes Rev. 2016;17(Suppl 1):62-67. doi:10.1111/obr.12381

52. Stranges S, Dorn JM, Shipley MJ, et al. Correlates of short and long sleep duration: a cross-cultural comparison between the United Kingdom and the United States: the Whitehall II Study and the Western New York Health Study. Am J Epidemiol. 2008;168 (12):1353-1364. doi:10.1093/aje/kwn337

53. Lauderdale DS, Knutson KL, Yan LL, Liu K, Rathouz PJ. Self-reported and measured sleep duration: how similar are they? Epidemiology. 2008;19(6):838-845. doi:10.1097/EDE.0b013e318187a7b0

54. Lo K, Woo B, Wong M, Tam W. Subjective sleep quality, blood pressure, and hypertension: a meta-analysis. J Clin Hypertens. 2018;20(3):592-605. doi:10.1111/jch.13220

55. Xiao L, Harrison DG. Inflammation in hypertension. Can J Cardiol. 2020;36(5):635-647. doi:10.1016/j.cjca.2020.01.013
Nature and Science of Sleep

\section{Publish your work in this journal}

Nature and Science of Sleep is an international, peer-reviewed, open access journal covering all aspects of sleep science and sleep medicine, including the neurophysiology and functions of sleep, the genetics of sleep, sleep and society, biological rhythms, dreaming, sleep disorders and therapy, and strategies to optimize healthy sleep.
The manuscript management system is completely online and includes a very quick and fair peer-review system, which is all easy to use. Visit http://www.dovepress.com/testimonials.php to read real quotes from published authors. 